158 Kl. Meng. Wass. i. Aether etc. - Nachw. d. Butters.etc. - Guttaperchafabr.

\title{
Um kleine Mengen Wasser im Aether zu entdecken,
}

verwendet J. Romei das phenylsaure Kali, welches gut getrocknet, in letzterem fast unlöslich ist, in wasserhaltigem Aether sich indess theilweise löst, während der ungelöst gebliebene Theil nach einiger Zeit eine rothbraune Farbe annimmt. Verfasser hat anf diese Weise in 1000 Thl. Aether noch 2,5 Thle. Wasser erkennen können. (Zeitschrift $f$. anal. Chemie 1869. 380.; daraus in Jahrb. f. Pharm. Bd. XXXIII. Heft 2. p. 85.).

C. Schulze.

\section{Nachweisung der Buttersåure im Glycerin und ihre Gewinnung daraus.}

Perutz versetzt, um zu erfahren, ob Glycerin Buttersäure-haltig ist, dasselbe mit starkem Alkohol und concentrirter Schwefelsäure, wo sofort Buttersäureäther auftritt, wenn Buttersäure zugegen ist.

Das këufliche Glycerin enthält nicht selten nahmhafte Mengen von Buttersäure, so dass deren Extraction lohnend ist; zu diesen Zwecke behandelt man die Thierkohle, welche zum Entfärben des Glycerins gedient hat und die die Buttersäure enthält, mit Weingeist, der den buttersauren Kalk aufnimmt und bei der Destillation zurücklässt. (Dingler's polyt. Journ. CLXXXVII. 258.; a. dems. in Wittstein's Vierteljahresschr. Heft 2. 1869. p. 298.).

C. Schulze.

\section{Guttaperchafabriken.}

Man schätzt die Anzahl von Guttaperchafabriken in Amerika und Europa auf 150, welche durchschnittlich jede 4-500 Leute beschäftigen. Diese Fabriken gebrauchen jëhrlich mehr wie 10 Millionen Pfd. Gutta. Trotzdem glaubt man, dass das ganze Geschäft noch in seiner Kindheit sei; sicher ist, dass es Tag für Tag wächst. Es ist vorlüufig keine Gefahr vorhanden, dass die Nachfrage aus Mangel an Rohmaterial nicht befriedigt werden könne. Der Gürtel Land, welcher in einer Breite von 500 engl. Meilen nördlich und 500 engl. Meilen südlich um den Aequator zieht, enthält eine sehr grosse Anzahl von Guttabäumen. Man kann dieselben 